\title{
Small-Signal Modeling of RF CMOS
}

\author{
Jaejune Jang, Robert W. Dutton* \\ Cypress Semiconductor, 3901 North 1st, San Jose, CA 95134-1599, USA, \\ jangj@gloworm.stanford.edu \\ *Center for Integrated System, Stanford University, Stanford, CA 94305, USA \\ robert.dutton@stanford.edu
}

\begin{abstract}
This paper presents accurate small-signal modeling of RF CMOS, valid from DC to GHz ranges, using device simulation and analytical modeling. Distributed NQS effects in terms of circuit parameters are discussed and an estimation of the limit up to which quasi-static MOSFET models are reasonable is presented. The impact of the substrate network through $\mathrm{g}_{\mathrm{mb}}$ multiplication on terminal ac characteristics is also discussed.
\end{abstract}

\section{Modeling and Analysis}

Calibration of the process simulator is done using SIMS to generate a two-dimensional device structure. IV characteristics are also calibrated using mobility parameters, Lombodi model, and suitable semiconductor workfunction. Once the process is calibrated, device structures with different channel length ( $\mathrm{L}=50 \mathrm{um}, 10 \mathrm{um}, 2.5 \mathrm{um}, 1.2 \mathrm{um}, 0.6 \mathrm{um}, 0.3 \mathrm{um}$, and $0.15 \mathrm{um}$ ) are generated using the process simulation. For each device, all sixteen yparameters, $\mathrm{y}_{\text {gd }}, \mathrm{y}_{\mathrm{gs}}, \mathrm{y}_{\mathrm{gb}}, \mathrm{y}_{\mathrm{dg}}, \mathrm{y}_{\mathrm{ds}}, \mathrm{y}_{\mathrm{db}}, \mathrm{y}_{\mathrm{sg}}, \mathrm{y}_{\mathrm{sd}}, \mathrm{y}_{\mathrm{sb}}, \mathrm{y}_{\mathrm{bg}}, \mathrm{y}_{\mathrm{bd}}, \mathrm{y}_{\mathrm{bs}}, \mathrm{y}_{\mathrm{gg}}, \mathrm{y}_{\mathrm{dd}}, \mathrm{y}_{\mathrm{ss}}$, and $\mathrm{y}_{\mathrm{bb}}$, are simulated for all bias regions $\left(0<\mathrm{V}_{\mathrm{DS}} \& \mathrm{~V}_{\mathrm{GS}}<3 \mathrm{~V}\right)$ from $10 \mathrm{~Hz}$ to $60 \mathrm{GHz}$.

The development of a small-signal model is based on the well known general four terminal y-parameter network as shown in Fig. 1a. The characteristic of each y-parameter in Fig. $1 \mathrm{a}$ is represented as a circuit form by analyzing the frequency response.

An often mentioned problem in RF CMOS modeling is non-quasi-static phenomena. It is reported that this NQS effect occurs most dominantly in $\mathrm{C}_{\mathrm{gg}}$ and $\mathrm{g}_{\mathrm{gg}}$ parameters as shown in Fig. 2 where gate capacitance, $\mathrm{C}_{\mathrm{gg}}$, degrades at high frequencies and the conductance increases proportionally to $f^{2}$, which is directly related to induced gate noise. However, NQS effects on bias dependency of individual modeling parameters (i.e., $\mathrm{C}_{\mathrm{gs}}, \mathrm{C}_{\mathrm{gd}}, \mathrm{C}_{\mathrm{sd}}$ etc.) are not well known. Fig. 3 shows the bias dependency of $C_{g s}$ and $C_{g d}$ for $L=1.2$ um device at frequencies of $6.5 \mathrm{GHz}$ and $17 \mathrm{GHz}$. In the linear region, the amount of degradation of $\mathrm{C}_{\mathrm{gs}}$ and $\mathrm{C}_{\mathrm{gd}}$ between $6.5 \mathrm{GHz}$ and $17 \mathrm{GHz}$ is the same but in the saturation region, $\mathrm{V}_{\mathrm{DS}}=\mathrm{V}_{\mathrm{GS}}=3 \mathrm{~V}$, the degradation of $\mathrm{C}_{\mathrm{gs}}$ is significant while $\mathrm{C}_{\mathrm{gd}}$ shows little degradation. This is due to the effective channel length, $\mathrm{L}_{e f f}$, of $\mathrm{C}_{\mathrm{gs}}$ and $\mathrm{C}_{\mathrm{gd}}$ which is bias dependent due to charge sharing as illustrated in Fig. 4. Thus the effective channel length is related as:

$$
L_{e f f-g s} \cong L \cdot \frac{C_{g s}}{C_{g s}+C_{g d}}
$$

For short channel devices, the NQS frequency, $f_{N Q S}$, where the capacitance starts to degrade can be formulated as: 


$$
f_{N Q S-C_{g s}}=n\left[\frac{\mu\left(V_{G S}-V_{T}\right)}{2 \pi L_{e f f}^{2}}\right]
$$

Where $\mathrm{n}$ is a fitting parameter. Similarly $f_{N Q S}$ for $\mathrm{C}_{\mathrm{gd}}$ can also be formulated.

The substrate network from drain and source terminals can be represented as parallel RC circuits, $R_{s}$ and $C_{s}$, as shown in Fig. $5 a$. This substrate network accurately represents the frequency response of source to bulk and drain to bulk. Extracted substrate parameters for both drain and source sides for all biasing regions are shown in Fig. 6. It is very interesting to note that the extracted substrate parameters, $\mathrm{R}_{\mathrm{S}_{-} \text {drain }}, \mathrm{R}_{\mathrm{S}_{-} \text {source }}, \mathrm{C}_{\mathrm{S}_{-} \text {source }}$, and $\mathrm{C}_{\mathrm{s}_{-} \text {drain }}$, show the following conservative behavior.

$$
R_{\text {s-drain }} C_{\text {s-drain }}=R_{\text {s-source }} C_{\text {s-source }}
$$

This relationship is due to the material property of the lossy silicon substrate, dictated by

$$
R_{s} C_{s}=\frac{\varepsilon_{s i} \varepsilon_{o}}{\sigma_{s i}}
$$

Simulated terminal capacitances of a long channel device, in Fig 7a, follow the same characteristics of intrinsic capacitances derived from the charge-based model. However, $\mathrm{C}_{\mathrm{ss}}, \mathrm{C}_{\mathrm{sg}}, \mathrm{C}_{\mathrm{sd}}$, and $\mathrm{C}_{\mathrm{sb}}$ for short channel device, in Fig. 7b, show significantly different characteristics (i.e., the capacitances are multiple values of maximum gate capacitance as high as 53 times). This is due to the existence of the substrate resistance and $\mathrm{g}_{\mathrm{mb}}$ as:

$$
C_{\text {sdeff }}=C_{s d}+\frac{R_{s u b} g_{m b}}{1+\omega^{2} R_{s u b}^{2} C_{b b}^{2}} C_{b d}
$$

likewise, the effective source to drain conductance is:

$$
g_{\text {sdeff }}=g_{s d}+\frac{\omega^{2} R_{s u b}^{2} g_{m b}}{1+\omega^{2} R_{s u b}^{2} C_{b b}^{2}} C_{b b} C_{b d}
$$

Where the $R_{\text {sub }}$ represents substrate resistance as shown in Fig. 5b. $C_{d s}, C_{d g}, C_{d s}$, and $C_{d b}$ show similar behavior. Since both $\mathrm{g}_{\mathrm{mb}}$ and $\mathrm{R}_{\text {sub }}$ are proportional to $1 / \mathrm{L}$, the $\mathrm{g}_{\mathrm{mb}}$ amplification (second term in Eq. 5 6) occurs increasingly for short channel devices. At high frequencies, the second term in Eq. 5 6 disappears and only intrinsic term remains. Fig. 8 shows that the intrinsic term $\mathrm{C}_{\mathrm{sd}}$, which is negative for long channel device at linear region, becomes positive as channel length decreases. It is reported [2] that this is caused by DIBL effects. This work shows that this dependency is mainly a geometrical effect as source and drain junction capacitance becomes dominent while intrinsic $\mathrm{C}_{\mathrm{sd}}$ decreases as $\mathrm{L}$ decreases.

For the common-mode configuration, the simple compact model accounting for the NQS effect, $\mathrm{g}_{\mathrm{mb}}$, and the substrate effect is developed, in Fig. 1b, resulting in error of less than $2 \%$ in terms of s-parameters for all biasing regions from $\mathrm{DC}$ to $\mathrm{GHz}$ range.

\section{References}

[1] Y. Tsividis, Operation and modeling of the mos transistor, McGraw-Hill, second edition, 1999.

[2] M. Je, H. Shin, SISPAD, 2003. SISPAD 2003, pp.247-250, September 2003. 


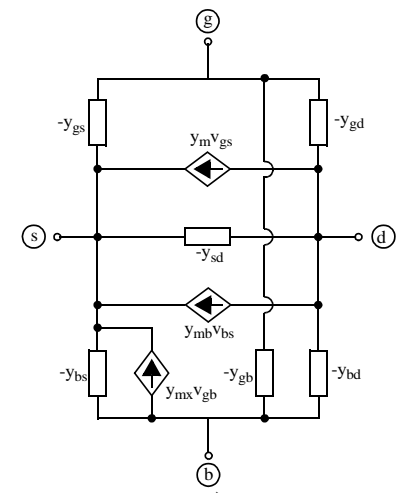

a)

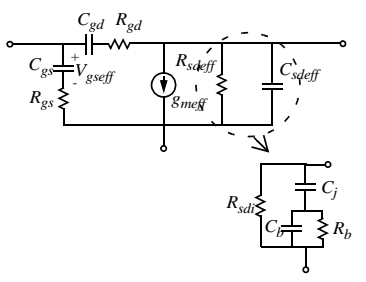

b)

Figure 1: a) general y-parameter network of four terminal MOSFET. b) Simplified small-signal model for common source configuration.
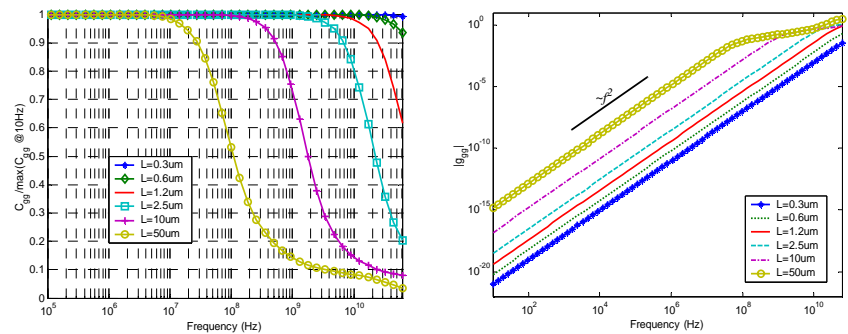

Figure 2: Simulated $\mathrm{C}_{g g}$ and $\mathrm{G}_{g g}$ vs. frequency for various channel length at $\mathrm{V}_{D S}=0$ and $\mathrm{V}_{G S}=3$ V. The $\mathrm{C}_{g g}$ is normalized by the maximum $\mathrm{C}_{g g}$ at $10 \mathrm{~Hz}$ for each device. Both $\mathrm{C}_{\mathrm{gg}}$ and $\mathrm{G}_{\mathrm{gg}}$ degrade at high frequencies with lower corner frequencies for long channel devices.

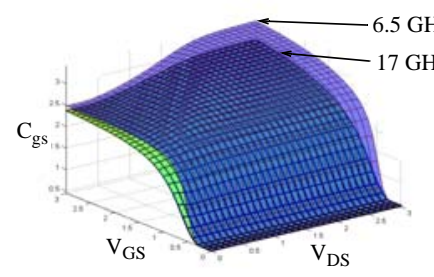

a) $\mathrm{C}_{\mathrm{gs}}$

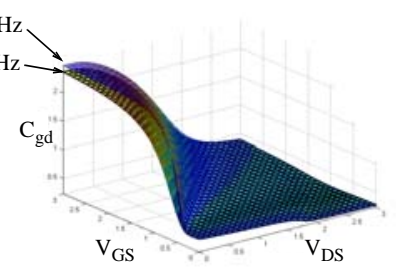

b) $\mathrm{C}_{\mathrm{gd}}$

Figure 3: Simulated a) $\mathrm{C}_{g s}$ and b) $\mathrm{C}_{g d}$ at $6.5 \mathrm{GHz}$ and $17 \mathrm{GHz}$ for $\mathrm{L}=1.2 \mathrm{um}$ for all bias region. In saturation region (i.e. $\mathrm{V}_{D S}=\mathrm{V}_{G S}=3 \mathrm{~V}$ ) the degradation of $\mathrm{C}_{g s}$ between 2 frequencies is higher compared to $\mathrm{C}_{g d}$, while the degradation of the $\mathrm{C}_{g s}$ and $\mathrm{C}_{g d}$ is similar in linear region.

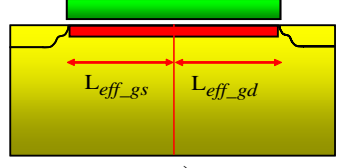

a)

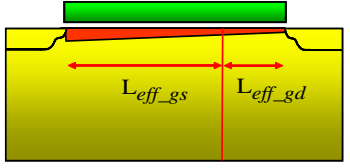

b)

Figure 4: a) when $\mathrm{V}_{D S}=\mathrm{V}_{S S}=0$ and $\mathrm{V}_{G S}>\mathrm{V}_{t h}$, inversion charge splits equally between drain and source resulting in $\mathrm{L}_{\text {eff_gs }}=\mathrm{L}_{\text {eff gd }}$, b) $\mathrm{V}_{D S}>\mathrm{V}_{S S}$ and $\mathrm{V}_{G S}>\mathrm{V}_{t h}$, source side shares most of charge resulting in $\mathrm{L}_{\text {eff_gs }}>\mathrm{L}_{\text {eff_gd. }}$ 

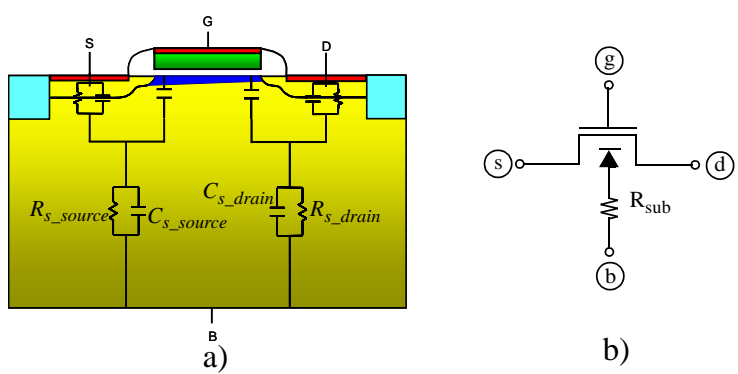

b)

Figure 5: a) substrate network of MOSFET and, b) simplified substrate network of MOSFET.

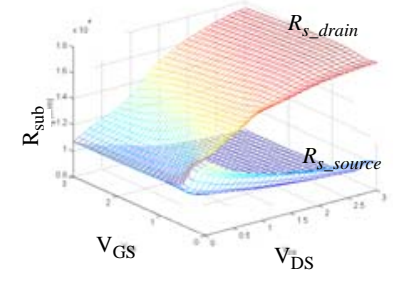

a)

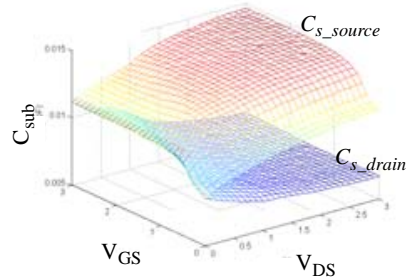

b)

Figure 6: Extracted substrate parameters of a) $\mathrm{R}_{\text {sub }}$ b) $\mathrm{C}_{\text {sub }}$.

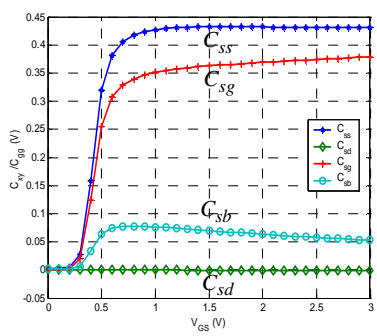

a)

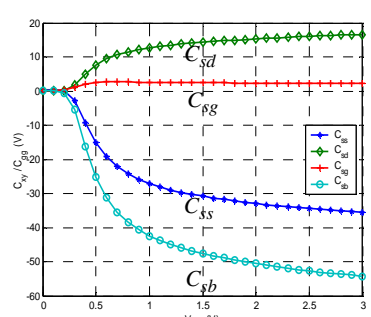

b)

Figure 7: Simulated $\mathrm{C}_{\mathrm{ss}}, \mathrm{C}_{\mathrm{sg}}, \mathrm{C}_{\mathrm{sd}}$, and $\mathrm{C}_{\mathrm{sb}}$ at $10 \mathrm{~Hz}, \mathrm{~V}_{D S}=\mathrm{V}_{G S}=3 \mathrm{~V}$ for a) $\mathrm{L}=10$ um device, and b) $\mathrm{L}=0.3$ um device.

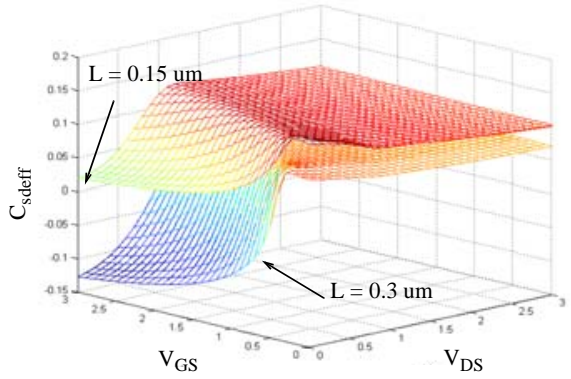

Figure 8: $\mathrm{C}_{\text {sdeff }}$ for $\mathrm{L}=0.15 \mathrm{um}$ and $\mathrm{L}=0.3 \mathrm{um}$ device at $10 \mathrm{GHz}$, where only intrinsic term, $\mathrm{C}_{\mathrm{sd}}$, remains. The $\mathrm{C}_{\mathrm{sd}}$ becomes positive for shorter channel length ( $\mathrm{L}=0.15 \mathrm{um}$ ) device. 